\title{
Lepton Number Violation in Low Scale Seesaw Mechanism and its Collider Complementarity
}

\author{
Prativa Pritimita*, Nitali Dash, Sudhanwa Patra \\ Center of Excellence in Theoretical and Mathematical Sciences, Siksha 'O' Anusandhan \\ University, Bhubaneswar-751030, India \\ E-mail: pratibha.pritimita@gmail.com
}

\begin{abstract}
We consider the $\mathrm{TeV}$ scale left-right symmetric theory which can accommodate low scale seesaw mechanisms consistent with neutrino oscillation data and find new physics contributions to neutrinoless double beta decay. The model facilitates natural type-II seesaw dominance and the presence of extra particles make the Dirac neutrino mass matrix $M_{D}$ large that leads to large light heavy neutrino mixing. The spontaneous symmetry breaking through doublets, triplets and bidoublet scalars at $\mathrm{TeV}$ scale offers rich phenomenology accessible to LHC. From the numerical studies of the new physics contributions to neutrinoless double beta decay we derive a lower limit on absolute scale of lightest neutrino mass and find that normal hierarchy $(\mathrm{NH})$ pattern is favorable taking into account the cosmology and oscillation data.
\end{abstract}

38th International Conference on High Energy Physics

3-10 August 2016

Chicago, USA

\footnotetext{
* Speaker.
} 
The origin of non-zero neutrino masses can be well-explained with low scale seesaw mechanisms that offer direct testability at LHC. Another low energy phenomenon that seems promising in the view that it can confirm Majorana nature of neutrinos is the neutrinoless double beta decay [1]. Interesting phenomenology can be expected when this process is studied in the framework of TeV scale left-right symmetric model (LRSM) [2,3]. We consider the extension of this model which comprises of usual quarks and leptons plus a sterile fermion per each generation and doublets, triplets, bidoublet scalars that help in the spontaneous symmetry breaking [4]. The gauge symmetry that governs the left-right symmetric model is $S U(2)_{L} \times S U(2)_{R} \times U(1)_{B-L} \times S U(3)_{C}$. When $S U(2)_{R} \times U(1)_{B-L}$ breaks at few TeV it leads to interesting collider signatures, neutrinoless double beta decay, lepton flavour violating processes and important implications to cosmology $[5,6,7,8,9,10,11,12,13]$. The neutral lepton mass matrix in the flavor basis of $\left(v_{L}, S_{L}, N_{R}^{c}\right)$ is given by

$$
\mathbb{M}=\left(\begin{array}{c|ccc} 
& v_{L} & S_{L} & N_{R}^{c} \\
\hline v_{L} & M_{L} & 0 & M_{D} \\
S_{L} & 0 & 0 & M \\
N_{R}^{c} & M_{D}^{T} & M^{T} & M_{R}
\end{array}\right) .
$$

where $M_{D}$ is the Dirac neutrino mass connecting $v_{L}-N_{R}, M$ is the $N_{R}-S_{L}$ mixing matrix and $M_{L}\left(M_{R}\right)$ is the Majorana mass for LH (RH) neutrinos. Using the mass hierarchy $M_{R}>M>M_{D} \gg$ $M_{L}$, the complete diagonalization procedure will lead to physical masses and mixing can be expressed in terms of light neutrino mass $m_{v}^{\mathrm{d}}=\operatorname{diag}\left\{m_{1}, m_{2}, m_{3}\right\}$ and oscillation parameters as

$$
m_{v}=U_{\mathrm{PMNS}} m_{v}^{\mathrm{d}} U_{\mathrm{PMNS}}^{T}, \quad M_{N}=\frac{v_{R}}{v_{L}} U_{\mathrm{PMNS}} m_{v}^{\mathrm{d}} U_{\mathrm{PMNS}}^{T}, \quad M_{S}=-m_{S}^{2}\left[\frac{v_{R}}{v_{L}} U_{\mathrm{PMNS}} m_{v}^{\mathrm{d}} U_{\mathrm{PMNS}}^{T}\right]^{-1}
$$

The extra particles substantially make the Dirac neutrino mass matrix $M_{D}$ large that allows large light-heavy neutrino mixing. The charge current interaction Lagrangian with the physical masses and mixing for neutrinos is given by

$$
\begin{aligned}
& \sum_{\alpha=e, \mu, \tau}\left[\frac{g_{L}}{\sqrt{2}} \bar{\ell}_{\alpha L} \gamma_{\mu} v_{\alpha L} W_{L}^{\mu}+\frac{g_{R}}{\sqrt{2}} \bar{\ell}_{\alpha R} \gamma_{\mu} N_{\alpha R} W_{R}^{\mu}\right]+\text { h.c. } \\
& \text { with } v_{\alpha}=V_{\alpha i}^{v v} v_{i}+V_{\alpha j}^{v N} N_{j}+V_{\alpha k}^{v S} S_{k} \\
& N_{\beta}=0 \times v_{i}+V_{\beta j}^{N N} N_{j}+V_{\beta k}^{N S} S_{k}+\text { h.c. }
\end{aligned}
$$

As an immediate result new contributions (displayed in Fig. 1) arise due to;

- purely left-handed currents via exchange of heavy right-handed neutrinos and sterile neutrinos,

- purely right-handed currents via exchange of heavy right-handed neutrinos and doubly charged scalar triplet,

- mixed helicity diagrams called as $\lambda$ diagrams involving light-heavy neutrino mixing.

- mixed helicity diagrams called as $\eta$ diagrams involving $W_{L}-W_{R}$ mixing. 

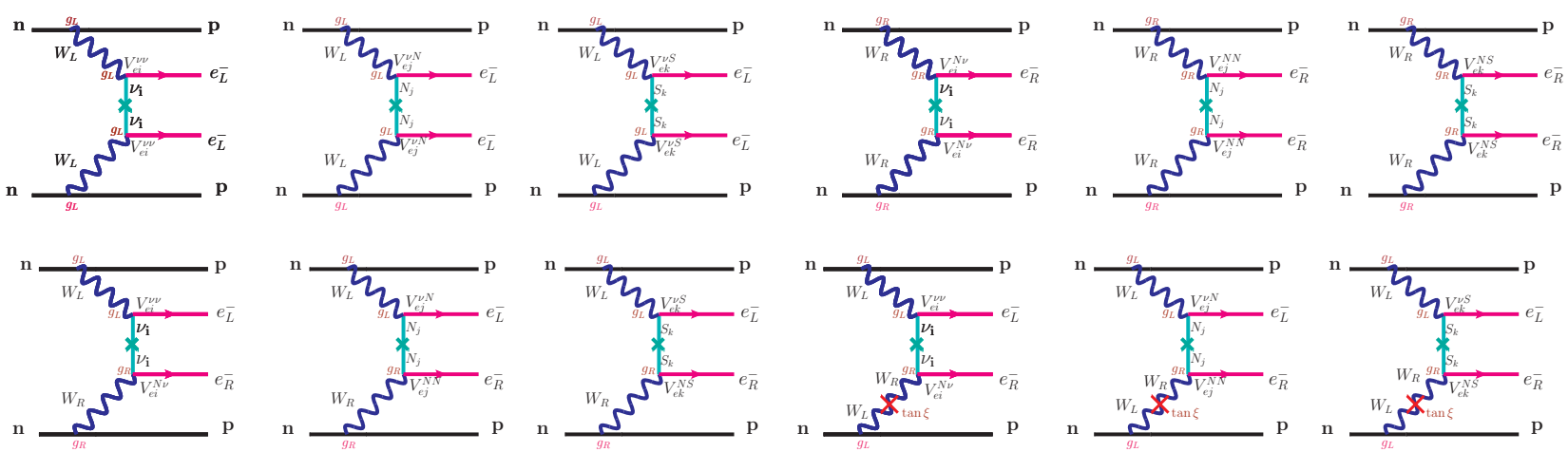

Figure 1: Feynman diagrams for neutrinoless double beta decay with $\mathrm{TeV}$ spectrum of $W_{R}, Z_{R}$ gauge bosons, heavy neutrinos.

We minutely analyze these new physics contributions and derive the absolute scale of lightest neutrino mass using cosmology [14] and oscillation data [15]. The calculations looks simple since we have expressed all the physical masses and mixing in terms of lightest neutrino mass and neutrino oscillation parameters. We derive the lower limit on the absolute scale of light neutrino mass by saturating the experimental limit from GERDA [16] and KamLAND-ZEN experiment [17] as displayed in Fig. 2.
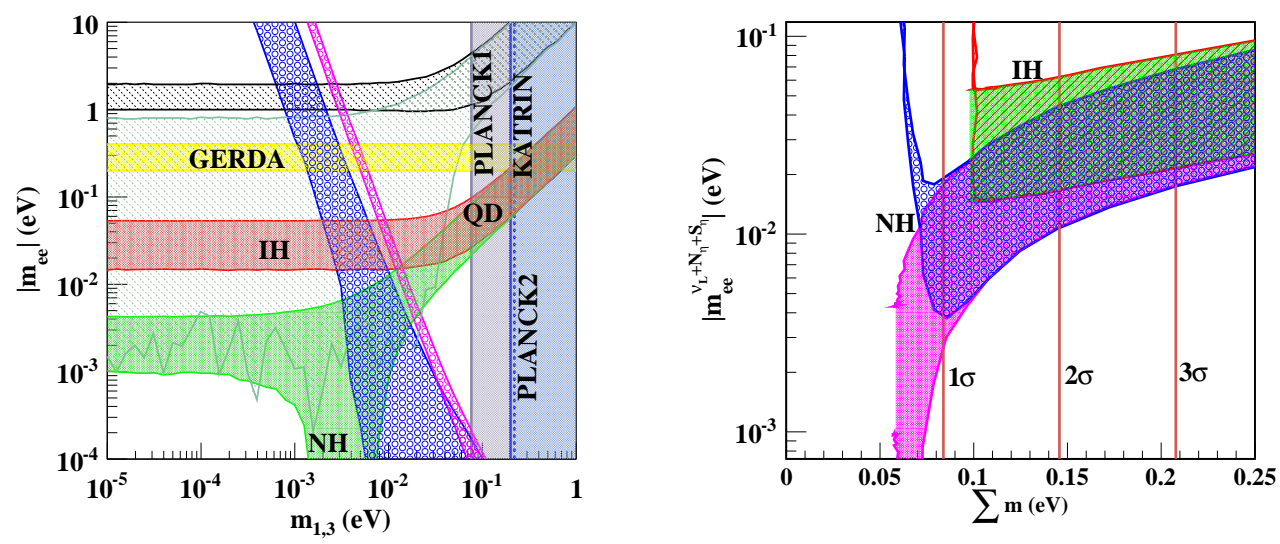

Figure 2: New Physics contributions to neutrinoless double beta decay within TeV scale LRSM. The leftpanel shows effective mass parameters vs lightest neutrino mass whereas the right-panel is for effective mass parameters vs sum of light neutrino masses.

We have discussed natural realization of type-II seesaw dominance within a TeV scale LRSM. We have expressed all the physical masses and mixings like $v_{L}, N_{R}$ and $S_{L}$ in terms of oscillation parameters and mass of lightest neutrino. We have derived the lower limit on absolute scale of 
lightest neutrino mass by numerically estimating new physics contributions to $0 v \beta \beta$ transition saturating the current experimental limit. The effective mass vs sum of light neutrino masses shows that $\mathrm{NH}$ pattern of light neutrinos is favorable.

\section{References}

[1] J. Schechter and J. W. F. Valle, Phys. Rev. D 25, 2951 (1982). doi:10.1103/PhysRevD.25.2951

[2] R. N. Mohapatra and J. C. Pati, Phys. Rev. D 11, 2558 (1975). doi:10.1103/PhysRevD.11.2558

[3] G. Senjanovic and R. N. Mohapatra, Phys. Rev. D 12, 1502 (1975). doi:10.1103/PhysRevD.12.1502

[4] P. Pritimita, N. Dash and S. Patra, arXiv:1607.07655 [hep-ph].

[5] J. Barry and W. Rodejohann, JHEP 1309, 153 (2013); [arXiv:1303.6324 [hep-ph]].

[6] V. Tello, M. Nemevsek, F. Nesti, G. Senjanovic and F. Vissani, Phys. Rev. Lett. 106, 151801 (2011); [arXiv:1011.3522 [hep-ph]].

[7] S. Patra, F. S. Queiroz and W. Rodejohann, Phys. Lett. B 752, 186 (2016); [arXiv:1506.03456 [hep-ph]].

[8] F. F. Deppisch, L. Graf, S. Kulkarni, S. Patra, W. Rodejohann, N. Sahu and U. Sarkar, Phys. Rev. D 93, no. 1, 013011 (2016); [arXiv:1508.05940 [hep-ph]].

[9] S. F. Ge, M. Lindner and S. Patra, JHEP 1510, 077 (2015); [arXiv:1508.07286 [hep-ph]].

[10] F. F. Deppisch, T. E. Gonzalo, S. Patra, N. Sahu and U. Sarkar, Phys. Rev. D 91, no. 1, 015018 (2015); [arXiv:1410.6427 [hep-ph]].

[11] R. L. Awasthi, M. K. Parida and S. Patra, JHEP 1308, 122 (2013); [arXiv:1302.0672 [hep-ph]].

[12] J. Chakrabortty, H. Z. Devi, S. Goswami and S. Patra, JHEP 1208, 008 (2012); [arXiv:1204.2527 [hep-ph]].

[13] J. Heeck and S. Patra, Phys. Rev. Lett. 115, no. 12, 121804 (2015); [arXiv:1507.01584 [hep-ph]].

[14] P. A. R. Ade et al. [Planck Collaboration], Astron. Astrophys. 571, A16 (2014); [arXiv:1303.5076 [astro-ph.CO]].

[15] M. C. Gonzalez-Garcia, M. Maltoni and T. Schwetz, JHEP 1411, 052 (2014); [arXiv:1409.5439 [hep-ph]].

[16] M. Agostini et al. [GERDA Collaboration], Phys. Rev. Lett. 111, no. 12, 122503 (2013); [arXiv:1307.4720 [nucl-ex]].

[17] A. Gando et al. [KamLAND-Zen Collaboration], Phys. Rev. Lett. 110, no. 6, 062502 (2013); [arXiv:1211.3863 [hep-ex]]. 